\title{
Advantages and disadvantages of distance learning on students' and teachers' of the physical culture faculty opinion
}

\author{
Alexey Korolkov ${ }^{1 *}$, Gennady Germanov ${ }^{2}$, Olga Langueva ${ }^{3}$, Arina Shevyakova ${ }^{1}$, Natalia Poskrebysheva $^{4}$ \\ ${ }^{1}$ Moscow State Regional University, 141014, Mytishchi, Russia \\ ${ }^{2}$ Russian State University of Physical Culture, Sports, Youth and Tourism, 105122, Moscow, Russia \\ ${ }^{3}$ Moscow State Pedagogical University, 119991, Moscow, Russia. \\ ${ }^{4}$ Belgorod University of Cooperation, Economics and Law, 308023, Belgorod, Russia
}

\begin{abstract}
We tryed to formalize various aspects of distance education in the form of speculative validity indicators based on calculating the relative frequency of students and teachers opinions. Methods of survey, questioning, systematization of information from open sources in electronic catalogs and publications on the Internet were used. The systematized nominal information was classified according to the selected dichotomous bases. There are 10 reasons (factors) for evaluating the advantages and disadvantages of distance learning. 26 aspects-variables of distance education were identified. Promising areas for further research are outlined.
\end{abstract}

\section{Introduction}

The self-isolation and distance learning in educational institutions because of the pandemic stimulate the use of elearning technologies in practice. The sudden transition to distance learning (DL) revealed all its advantages and disadvantages, which are discussed by the pedagogical community in scientific publications and on the Internet. The transition to DL can be considered as a transition of the education system to a new state - a state of crisis characterized by the disappearance of a certain number of elements of the system and a change in its integral characteristics. The transformation of the education system was the result of an extraordinary adaptation of the system to the conditions of the pandemic.

Physical contacts between the process participants completely stopped, paper document management operations and most operations related to the transmission of analog information in the form of written and oral speech disappeared. The volume of information exchange in electronic digital form has increased: email, messengers, social networks, special communication and educational platforms: zoom, teams, mirapolis, moodle, skype, etc. Due to the isolation regime, the ways of information exchange in the education system were redistributed. Duplicating bureaucratic actions disappeared, and the information redundancy of labor operations decreased. By analogy with the restriction of the living organisms' sensory systems, the compensatory mechanisms of other subsystems that duplicate the channels of information exchange become more acute. In our case, the main one is the remote electronic information exchange method. Such restriction of other information exchange methods, as in any other complex systems, reduces the effectiveness of the educational system to achieve its goal.

It is relevant to consider the advantages and disadvantages of the program, both on the part of teachers and students. At the same time, teachers, especially those of the older generation, were often less prepared to switch to distance learning than students, belonging to the so-called "Z" generation. They developed electronic communication skills.

\section{Materials and methods}

To solve this problem, in may-June 2020, a remote survey was conducted of 58 second-year students of the Moscow State Regional University physical culture faculty. They randomly determined one main advantage and one main disadvantage of DL.

According to the teachers, the positive and negative aspects of DL were evaluated as a result of the publications analysis for 2019-20 that are publicly available in the e - library [1, 2, 6, 7, 9-16] and information on the Internet [8].

The obtained data on the advantages and disadvantages of the system were systematized. The frequency of different responses was calculated. The general grounds for the development of psychometrically reliable questionnaires and the formalization of data suitable for processing by mathematical methods were determined.

\section{Results and discussion}

\footnotetext{
${ }^{*}$ Corresponding author: korolkov07@list.ru
} 
The results of any study depend on the composition and completeness of the initial data. This is especially evident in mass sociological and psychological research. In it the sociometric and psychometric reliability of research is often not evaluated: the results of research are accepted as unconditionally correct, unique and final [3]. Therefore, the results of studies of the same phenomenon or process conducted on different source data often do not coincide or contradict each other. For this reason, it is very important to determine the most complete set of initial data for studying a phenomenon, including the phenomenon of distance learning.

According to 58 students' opinion, the advantages and disadvantages of DL are divided into 4-5 groups. They can serve as a starting point for further classification of DL aspects.

A characteristic feature of the advantages (72\%) noted by students are the FEATURES that directly affect their personal life and convenience: saving time and money (41\%) and comfortable learning conditions (31\%). It is clear that these aspects have an invariant relation to the process and content of training. Only $21 \%$ of the students ' responses showed such a positive property of DL as flexibility in planning training and the ability to master new courses. We can assume that this percentage characterizes the number of students with high motivation to study, regardless of its implementation form.

The comfortable living conditions in home environment was never noted in the assessments of teachers.

The students attributed to disadvantages of DL a lack of live communication with fellow students and teachers, technical problems and an increase in the academic load. This data is quite consistent with the results of a study conducted on a sample of 35,000 students.

The negative aspects of DL noted by students relate primarily to the direct impact on their personal life and are not related to the content and quality of the learning process.

As a result of analyzing the publications of many researchers, other aspects of DL are also identified. They can be classified according to different dichotomous grounds (table 1). Each of them can be attributed to one or another aspect with different speculative validity. In different studies these grounds can be evaluated as positive, indicating the benefits, and negative, or mentioned only in one context.

Table 1. Dichotomous bases of DL aspects

\begin{tabular}{|c|l|l|}
\hline №oo & Advantages & Disadvantages \\
\hline 1. & distance - contact & isolation \\
\hline 2. & savings - costs & problem- solution \\
\hline 3. & processability - randomness & impact-ignoring \\
\hline 4. & Impact-indifference & theory-practice \\
\hline 5. & & \\
\hline 6. & flexibility - the inertia & requirement-leniency \\
\hline 7. & & a problem - help \\
\hline 8. & discipline - anarchy & \multicolumn{2}{|l}{} \\
\hline 9. & simplicity - complexity
\end{tabular}

The reasons given in table 1 be called factors of DL. They can be correlated with certain aspects-variables listed by different researchers with different frequency. The frequency of mentioning a particular DL aspect characterizes the speculative validity of this aspect. By analogy with confirmatory factor analysis, the frequency of mentioning each aspect (variable) can be considered as a kind of factor load - the correlation coefficient between the base (factor) and the aspect (variable).

There were at least 26 aspects (nominal variables) determined. These aspects are formulated differently by different researchers. But they have the same semantic meaning. The ratio of positive and negative aspects the same $58 \%$ and $42 \%$.

Researchers define the factor of distance-contact (isolation-unity) by such positive variables as: geographical freedom [1,9,12] and unlimited audience coverage [6]. It is also noted that distance learning is most convenient for part-time students, students of high-class athletes and other students with limited mobility [16].

The disadvantages of the distance aspect include: lack of personal contacts between the teacher and students $[1,17$, $15,11,2,4]$, lack of contacts between students [15], problems with cheating and identification of students [1, 9, 2], lack of direct control [9, 15], lack of students individual characteristics consideration [16]. The negative impact of the isolation factor can also be attributed to a separate aspect of the practical skills development: the low level of practical skills formation and the inability to master some special disciplines [1], the lack of practical training [2, 9].

As many teachers note, the lack of social contact and practical training negatively affects the educational component of education. In the distance format, the direct influence of the teacher on the student is completely eliminated, the value of the teacher's personal example is reduced, and the possibility of transmitting academic ethics and learning traditions disappears [8].

The learning flexibility factor is made up of only positive aspects-variables. They include: the possibility of combining their professional activities, self-scheduling and intensity of training loads [1, 15], the modul structure of courses and the parallel learning possibility [6], the individuality of learning, efficiency of communication with the teacher, the opportunity to combine courses [17], the possibility of personal improvement and knowledge management, expanding horizons [12], flexible scheduling of learning [9]. 
The processability factor is made up of both positive and negative aspects. Positive aspects include: objectivity of educational achievements assessment [1,2], consistency of modular course construction [16], the need to learn new technologies [13]. Negative components of this factor refer to various technical problems: availability and speed of Internet connection, and characteristics of the computer structure and design e-course, instructional course development, problem identification $[1,9,10,11,2,13]$. In both cases, the implementation of the technology factor requires high motivation and discipline of the student $[13,2,1]$.

Technologicality of DL places special requirements on the activity of the teacher. The teacher is forced to systematize the training material, present it in a formalized view, develop tests, presentations, training videos, and perform other necessary actions to present the training course in a digital educational environment.

Cost-effectiveness is an obvious factor of DL. It is to save time and costs for moving to the place of training, as well as to save nervous energy in stressful situations typical of full-time training [1]. The negative aspects of costeffectiveness include time constraints when completing tasks [11], increasing the load on students and teachers [14, 10, 13]. In this case, some teachers often overload students with independent tasks instead of preparing electronic teaching materials.

The influence factor is made up of two aspects: the direct influence of DL on the personalities of students and teachers, and the personal requirements necessary for the successful implementation of DL. Positive aspects include increased discipline and activity of students, the inevitability of mastering new technologies, increased independence and general outlook of students, the ability to independently study new courses, increased interest in learning, etc. [15, 13]. The negative aspects of this factor include: lack of systemic thinking, there is a load on the visual channel of perception and discrimination auditory and kinesthetic channels, the lack of speech development, violation of requirements to the limitations of continuous work on the computer [5, 20], increasing physical inactivity, high level of self-discipline and motivation, replacement of group sessions and individual-independent $[15,14,7]$.

\section{Conclusions}

As a result of the conducted research, it was found that DL has many advantages and disadvantages that relate to each other in approximately equal proportions. For this reason, DL cannot be the only and final form of training. According to students and teachers opinion, the main drawback of DL is the lack of social contact between participants in the educational process. It is necessary to ensure the educational component of the pedagogical system. That's why, distance learning is only suitable for students with a high degree of motivation to learn.

A significant disadvantage of DL is the inability to conduct practical classes under the direct supervision of a teacher. It negatively affects the development of practical skills in practice-oriented academic disciplines, including medicine and physical education.

Thus, this form can only be considered as an additional useful tool in the system of teaching physical culture. Distance learning can be recommended for use when conducting theoretical classes with senior students and undergraduates who have already mastered practical skills in physical education and for students with limited mobility in space.

The development of self-assessment methods for teachers and students on the dichotomous grounds is a promising area of research on the DL aspects.

\section{References}

1. I.V. Abdrakhmanova, V.A. Balueva, I.V. Lushchik, Physical education and sports training, 4(30), 119-125 (2019)

2. A.V. Ageyevets, V.Yu. Efimov-Komarov, L.B. Efimova-Komarova, E.A. Nazarenko, M.V. Puchkova, Scientific notes of the University named after P.F. Lesgaft, 4(182), 3-9 (2020)

3. A.S. Kuznetsov, Z.M. Kuznetsova, Russian Journal of Physical Education and Sport, 14(4), 5-7 (2019). DOI: 10.14526/20704798-2019-14-4-5-7

4. G.N. Germanov, L.A. Novikova, M.Yu. Sekovanich, A.N. Korolkov, A.P. Strizhak, Scientific notes of the University named after P.F. Lesgaft, 10(176), 82-91 (2019)

5. Hygienic requirements for personal electronic computers and organization of work (SanPiN 2.2.2/2.4.1340-03)

6. O.U. Digtyar, World of science, culture, education, 5(78), 342-344 (2019)

7. V.U. Ziambetov, D.V. Brovikov, Academic journalism, 4, 455-459 (2020)

8. S. Kislitsyn, Professor: distance learning is a profanation of education (URL: https://rossaprimavera.ru/article/3dabcedf)

9. S.V. Kochetova, International Journal of Humanities and Natural Sciences, 9-1, 32-34 (2019)

10. V.N. Kremneva, L.A. Nepovinnykh, E.M. Solodovnik, Questions of pedagogy, 6-1, 184-188 (2020)

11. T.U. Krestyaninova, O.N. Malakh, Bulletin of Omsk State Pedagogical University. Humanities research, 1(22), 108-111 (2019)

12. O.V. Kuzhelnaya, A.M. Atayan, A.M. Smirnov , D.G. Petrova, Scientific notes of the University named after Lesgaft P.F., 9(175), 151-153 (2019)

13. T.L. Kuzmenkova, Scientific notes of the University named after Lesgaft P.F., 2(180), 191-195 (2020)

14. M.M. Milko, N.V. Guremina, Modern high-tech technologies, 5, 195-200 (2020)

15. A.A. Popova, I.A. Nazarov, Sustainable development of science and education, 4, 348-353 (2019) 
16. N.V. Popova, Current scientific research in the modern world, 2-5(46), 144-148 (2019) 